\title{
A PROGRAMMED SPOT AUTOMATIC SCANNING SYSTEM FOR SPARK CHAMBERS*
}

\author{
A. SAULYS, D. I. MEYER and R. ALLEN
}

Physics Department, University of Michigan, Ann Arbor, Michigan

Received 16 August 1965

A relatively simple system has been built for automatically scanning any rectangular array of spark chamber views on film. It has been successfully used to scan an experiment with 24

\section{Introduction}

In a spark chamber experiment where a large number of photographs are taken, an automatic film scanning system reduces the analysis time by a considerable amount. For example, it takes about ten minutes for a human scanner to measure a typical 3 track spark chamber event. In an experiment on which the system has just successfully been used, 24 spark chamber views per frame were scanned and measured at the rate of one frame per second, with successful handling of 90 per cent of the events.

The basic feature of the device which distinguishes it from other systems ${ }^{1-3}$ ) is the balance which has been struck between the ability to handle many different spark chamber arrays and the complexity of a system needed to do this. In striking this balance there were several points which were considered.

First, the physics of an experiment only occassionally dictates the use of other than a rectangular array of chambers. Having the chamber plates parallel and perpendicular to the direction of the film travel simplifies the programming considerably.

Second, while speed is not usually a serious factor in an automatic system an area scan of an entire picture is time consuming and has the additional disadvantage that much of the information gathered is useless and must be immediately eliminated by the computer to avoid clogging the computer memory. The other extreme of finding a track, then following it through the spark chamber array, has only limited flexibility and could require considerable reprogramming from one experiment to the next. The approach used here was to program to handle any rectangular array of chambers, but to scan only the center of the spark chamber gaps (the position of the gaps can be fed in as auxiliary data at the beginning of each experiment). This limited area scan has no disadvantages over a full area scan and takes a length of time comparable to track following ${ }^{1}$ ).

* Work supported by the U.S. Atomic Energy Commission and the Office of Naval Research. views at the rate of about one frame per second. The optics, electronics, programming and the principles of operation are discussed.

The system uses the light from a programmed spot on a cathode ray tube to find the spark center. Tracks are then reconstructed from the coordinates found for the centers of each spark. The sparks are fit to tracks by choosing a series of small areas of each spark chamber and best fitting all sparks in the region to a straight line. (Some sophistication is added to this to handle delta rays and other spurious sparks). The shape and size of these areas can be easily changed for each experiment.

\section{Description of the system}

The hardware of the system consists of a cathode ray

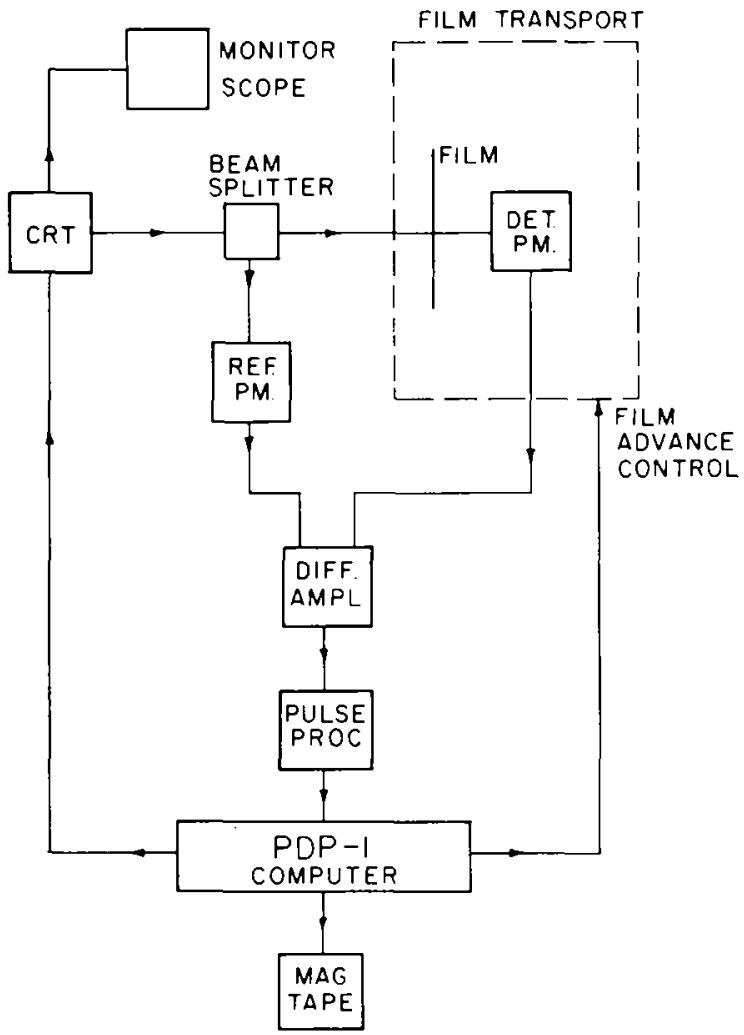

Fig. 1. Block diagram of the system. 
display tube under the control of a small computer (PDP-1 manufactured by the Digital Equipment Corporation), a film transport and the associated optics (fig. 1). The PIPP-1 computer controls the position of the point displayed on the cathode ray tube, receives the information as to whether that point is clear or dark on the film, controls the advance of the film to the next frame and writes the data on magnctic tape. The cathode ray display tube is type $30 \mathrm{~K}$, manufactured by the Digital Equipment Corporation. It has a square grid of $1024 \times 1024$ points which can be displayed in any order under the control of the computer. Points can be displayed at a maximum speed of $25 \mu \mathrm{s} \mathrm{per}$ point. The film transport was designed and built at the Lniversity of Michigan. It has the capacity of handling rolls of film up to 1000 feet in length and advances a frame of film in $100 \mathrm{~ms}$, including the time taken to unclamp one frame and clamp the next into position.

The optics are outlined in fig. 2. The light from a particular point on the cathode ray tube is split by the first beam splitter into a reference photomultiplier and a second beam splitter. The second beam splitter allows for another film transport so that if an event from an experiment is on two films the two frames can be scanned consecutively. The light from the second beam splitter is focused on the film by the imaging lens. The transmitted light is then detected by a photomultiplier behind the film. The field lens located between the film and the photomultiplier collects all of the light from any point on the film onto a diffuse circle of about $1 \mathrm{~cm}$ in diameter on the photocathode of the photomultiplier. This averages over any irregularities of the sensitivity of the photocathode. Thus there is a difference in the signal of the two photomultipliers where ever a dark point is found on the film.

It was found to be very useful to be able to view the frame that is being scanned. This was accomplished by moving a mirror into position at 45 behind the lens (fig. 2). When the projection lamp in front of the mirror is turned on, the field lens acts as a condensing lens and the imaging lens as a projection lens. The light is reflected by the second beam splitter and a series of mirrors onto a vicwing screen where the image of the frame that is being processed can be viewed.

Most of the electronics necessary for the system is part of the computer and the cathode ray tube. Additional electronics is necessary only to process the signals from the two photomultipliers (type 6810A). Every time a point on the cathode ray tube is intensified and the difference between the signals of the two photomultipliers increases past a preset level, the computer is interrupted indicating a dark point on the film. This is accomplished by a difference amplifier. The threshold between clear and dark is set by adjusting the high voltage on the photomultiplier dynodes. The film advance is actuated by means of a relay output on the computer. An additional piece of electronics equipment that was found very useful was a Tektronix type 503 oscilloscope as used by Deutsch ${ }^{1}$ ). It is connected in parallel with the scanning tube and thus the action of the scanning tube can be observed.

\section{Computer programs}

In scanning spark chamber photographs a line of points is displayed along the center of each gap to locate the sparks. Once the centers of all the pertinent dark objects (i.e. fiducial marks, sparks and binary frame numbers) are found they are ready for processing into tracks. Because of the limited memory of the PDP-1 the coordinates are written on magnetic tape which is processed on an IBM 7090 computer.

The programs were written as generally as possible

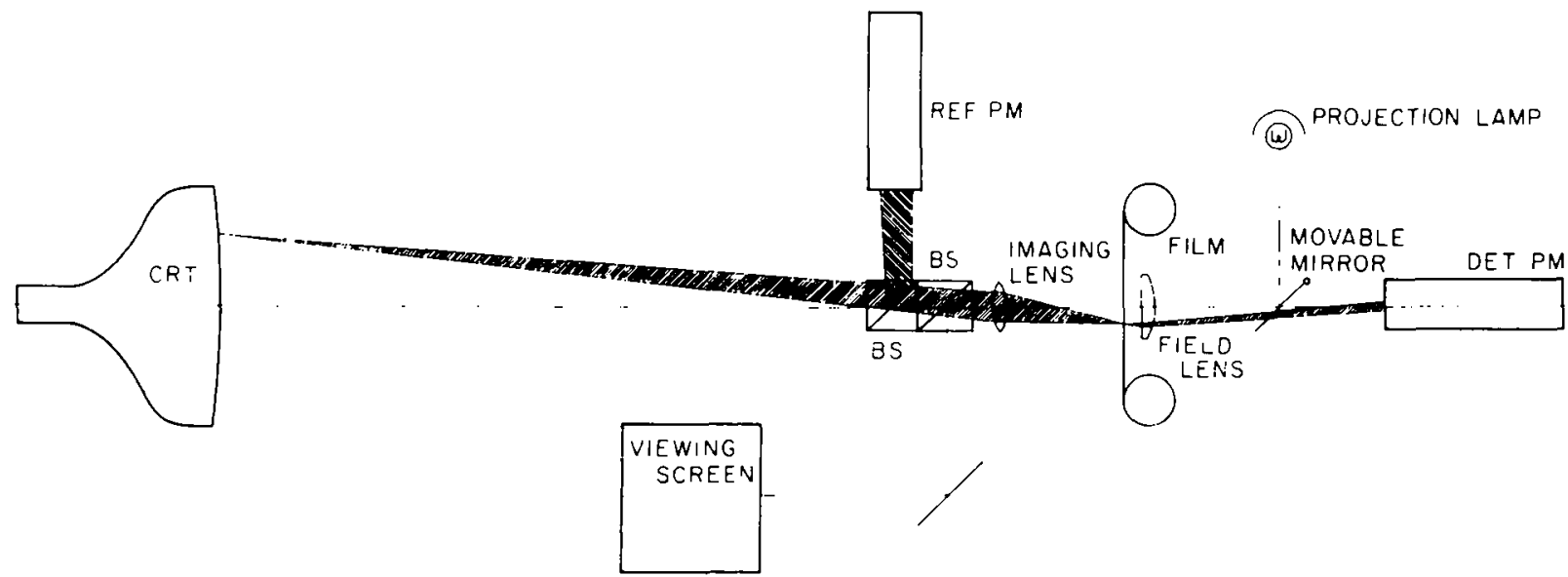

Fig. 2. Schematic diagram of the optics. 
without making them too cumbersome, so they could be used for any experiment with a rectangular array of spark chambers. The logic of the programs is split into two main parts: Scanning for sparks and track finding. The former is done on the PDP-1 computer and the latter on an IBM 7090.

In addition to the scanning program, a calibration program is used on the PDP-1. In order to scan only certain parts of the film, it is necessary to define these by means of a numerical table. Since each experiment has a different layout, this table has to be prepared for cach experiment by the calibration program. The table consists of the locations of spark chamber gaps, fiducial marks and any other markings on the film (e.g. frame number). These locations can be obtained by two methods. One way is to measure the positions on any hand measuring machine, scale the coordinates and feed them to the calibration program which will then assemble the table into the form required by the scanning program. Another way is to scan the entire frame of film, display it on the Tektronix monitor scope, use a movable pointer (also displayed) to point to the object whose location is desired and request the program to find the center. Its center is calculated and entered into a table. Thus by either of these two methods a table of the search areas is prepared and punched out on paper tape by the calibration program.

The scanning program looks for dark objects in the locations specified by the table prepared by the calibration program. Whenever it finds a dark object it locates both edges and calculates a center. The program searches for and must find another center just above and below the original line of search. Thus the requirements for something to be recognized as an object are three centers perpendicular to the direction of search and of specified width. This is sufficient to distinguish sparks from background with a high degree of reliability. When a search of the area specified in the calibration table is completed, the average value of the three centers found in the three scans of an object is written on magnetic tape. One has the option to display at any time on the monitor oscilloscope the objects found in scanning a frame of film. This greatly facilitates the adjustment of the threshold for dark objects and the locating of difficulties in scanning (fig. 3).

The coordinates of all the sparks and fiducial marks written on magnetic tape must now be put together into tracks. This is done by the trackfinding program on the IBM 7090 computer. The basic logic of the program is as follows. Each spark chamber view is subdivided into small sections around each spark found in that view. If there is a sufficient number of sparks in that section to form a track and no more than one spark per gap, then the sparks are least squares fitted to a straight line. If there is an insufficient number, then the next section is tried until all the sparks in that view are exhausted. If there is more than one spark per gap in a particular section, then all the possible combinations of the sparks are tried and the one that fits a straight line best is kept. Delta rays will sometimes rob a spark from the true track. This effect appears as a displaced spark. The program logic eliminates this by looking at the deviations of the sparks from a straight line and throwing away any sparks whose deviation is cxcessive, so only sparks which fit a straight line well are used. After all the sparks in all the individual views are fitted to subtracks then they are assembled into tracks by grouping all the subtracks with similar angles. If there is an appropriate number of tracks to form an event of interest, then the coordinates of each track in each view are written on magnetic tape to be processed by the spacial reconstruction and kinematics program.

\section{Measurement errors}

To obtain the full use of the accuracy of the cathode ray tube, some corrections to the coordinates of the objects found by the scanning system are necessary. First, the cathode ray tube has pincushion distortion which could possibly be optically corrected. But since there is some distortion inherent in the lenses and the beam splitters, the correction is done by the computer after the objects are found. The distortion was measured by inserting a piece of unexposed film in the position where the film to be scanned is placed and intensifying every 32 point on the cathode ray tube. In this way a photograph of the raster of points after the light goes through all the optics was obtained. The positions of the points were measured by hand on a measuring machine which has a resolution of about 7 times that of the automatic scanner. A functional form was obtained for the deviation of those points from a square raster. By the use of these functions the computer corrects for all optical distortions.

Since the resolution of the cathode ray tube is only 1024 points and the reproducibility is about $1 / 2000$, it is possible to measure the position of an object better than the tube resolution by measuring the position of the object at several places. In practice it is found that measurements (as determined by the consistency of the origin of two prong events) of spark positions are made to an rms accuracy of $1 / 2000$.

The 10 per cent of the events which are not handled automatically are largely due to poor firing of one of 


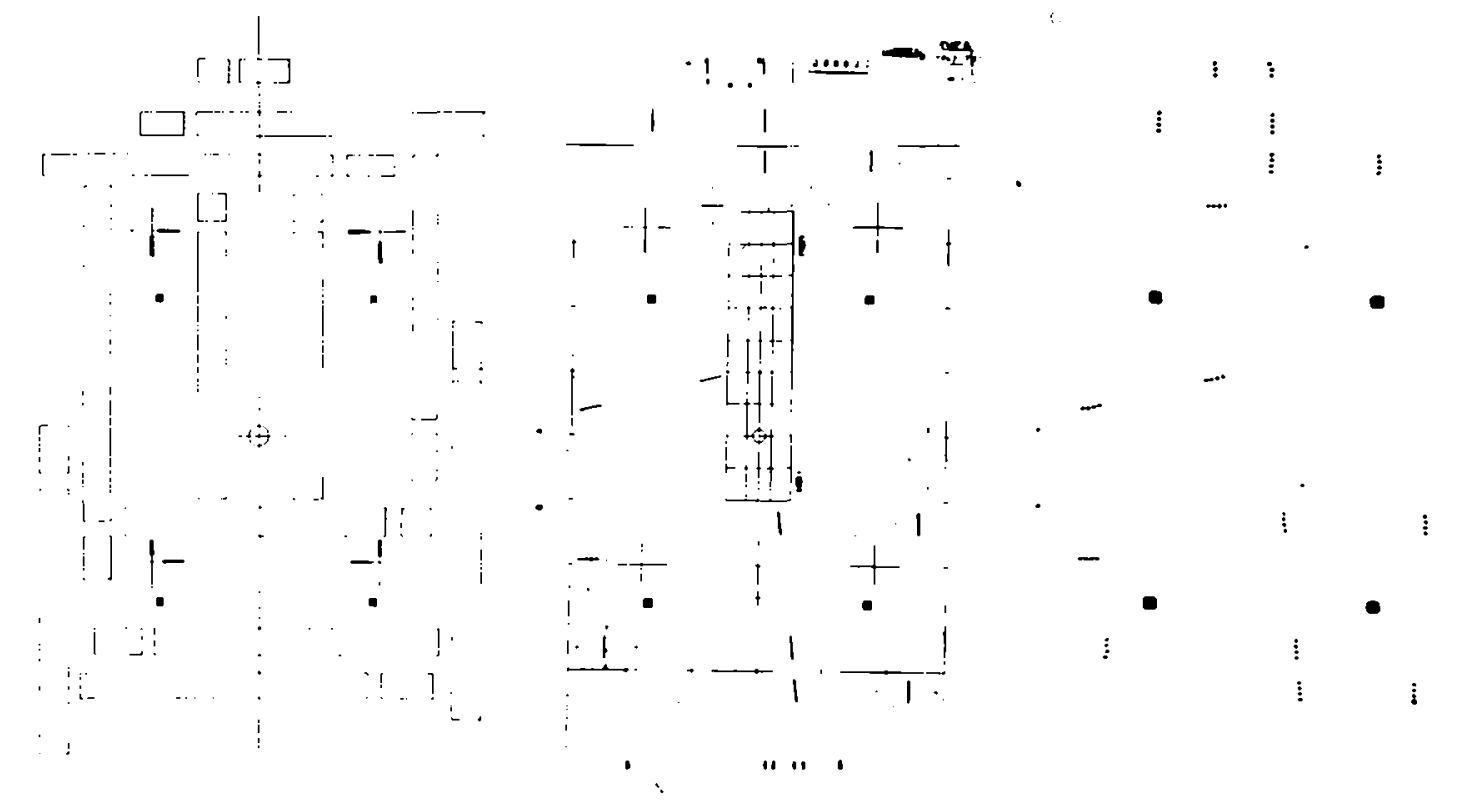

Fig. 3. Left: A layout of the spark chamber views of the experiment, which the system is presently scanning. The small rectangles are the end views. Center: A photograph of a typical event. A hydrogen target is in the grid area. Right: The same event played back on the monitor scope after it has been scanned by the system.

the spark chambers. By more complex programming, a large fraction of these as well as most events rejected for other reasons could be handled. This programming would have to be specialized to a particular experiment, however and does not seem worth the effort.

Instead, an alternative approach has been developed which semi-automatically untangles these events. The sparks found by the automatic scanner are displayed on a type 340 scope (manufactured by Digital Equipment Corporation). A light pen attached to the computer is then used to pick out the sparks which actually belong to the event. The rest of the sparks are then rejected and the analysis of the events proceeds as before. It has been found that this technique is 5 times as fast as hand digitizing and that only about 2 per cent of the events are left to be hand digitized. These consist of events with light tracks which cannot be distinguished from background with the automatic scanner.

\section{Conclusions}

Since the automatic scanner only finds the points on the film either dark or clear, differentiation of real objects from background noise is much better if the objects are much darker than the background and are of reasonable size compared to the resolution of the cathode ray tube. This must be kept in mind when the experiment is designed so that the problems of pattern recognition do not become overwhelming. For example, the fiducial marks must be larger and darker than any scratches or dirt on the film. For this purpose, electroluminescent panels were found to be ideal ${ }^{4}$ ).

A great deal of care should be taken to have efficient spark chamber operation. It is also desirable to have more redundancy in the number of gaps required to identify a track than when using hand measuring. Greater care on these 2 points would have decreased the number of events which the automatic system could not handle to just a few per cent.

We would like to acknowledge the work of K. Fauser and P. Wilcox on computer programming and $\mathrm{P}$. Goebel for help on the mechanical and optical system.

\section{References}

1) H. Rudloe, M. Deutsch and T. Marill, Communications of the ACM 6 (1963) 332.

2) Conference for programming for flying spot devices, CERN, 65-11 (1965).

3) L. M. Blair et al., Nucl. Instr. and Meth. 27 (1963) 93.

4) A. Saulys and E. Meanley, Rev. Sci. Instr. 36 (1965) 1053. 